\title{
MST1 Is a Multifunctional Caspase-Independent Inhibitor of Androgenic Signaling
}

\author{
Bekir Cinar ${ }^{1,2}$, Filiz Kisaayak Collak ${ }^{1,2}$, Delia Lopez ${ }^{3}$, Seckin Akgul ${ }^{4}$, Nishit K. Mukhopadhyay ${ }^{3}$, \\ Murat Kilicarslan ${ }^{5}$, Daniel G. Gioeli ${ }^{6}$, and Michael R. Freeman ${ }^{3}$
}

\section{Abstract}

The MST1 serine-threonine kinase, a component of the RASSF1-LATS tumor suppressor network, is involved in cell proliferation and apoptosis and has been implicated in cancer. However, the physiologic role of MST1 in prostate cancer (PCa) is not well understood. Here, we investigated the possibility of a biochemical and functional link between androgen receptor (AR) and MST1 signaling. We showed that MST1 forms a protein complex with AR and antagonizes AR transcriptional activity as shown by coimmunoprecipitation (co-IP), promoter reporter analysis, and molecular genetic methods. In vitro kinase and site-specific mutagenesis approaches indicate that MST1 is a potent AR kinase; however, the kinase activity of MST1 and its proapoptotic functions were shown not to be involved in inhibition of AR. MST1 was also found in AR-chromatin complexes, and enforced expression of MST1 reduced the binding of AR to a well-characterized, androgen-responsive region within the prostate-specific antigen promoter. MST1 suppressed PCa cell growth in vitro and tumor growth in mice. Because MST1 is also involved in regulating the AKT1 pathway, this kinase may be an important new link between androgenic and growth factor signaling and a novel therapeutic target in PCa. Cancer Res; 71(12); 4303-13. (C)2011 AACR.

\section{Introduction}

The serine-threonine kinase MST1 or STK4 (mammalian sterile STE20-like kinase 1), a homolog of Hippo (Hpo/hpo) in Drosophila, was originally identified as a proapoptotic protein (1). MST1 is related to 3 paralogs (MST2, MST3, and MST4) with a conserved structure consisting of $\mathrm{N}$-terminal catalytic (MST1-N) and C-terminal regulatory (MST1-C) domains and other functional sites, including caspase cleavage sites and nuclear export signals $(2,3)$. MST1 or MST2 can be activated by autophosphorylation of a unique threonine residue (Thr183 in MST1 and Thr-180 in MST2) in the activation loop or by

\footnotetext{
Authors' Affiliations: ${ }^{1}$ Departments of Medicine-Hematology/Oncology and Biomedical Sciences, Samuel Oschin Comprehensive Cancer Institute, Cedars-Sinai Medical Center; ${ }^{2}$ Department of Medicine, David Geffen School of Medicine, University of California, Los Angeles, California; ${ }^{3}$ Urological Diseases Research Center, Children's Hospital Boston; Departments of Surgery and Biological Chemistry and Molecular Pharmacology, Harvard Medical School, Boston, Massachusetts; ${ }^{4}$ Bilkent University, Bilkent, Ankara, Turkey; ${ }^{5}$ University of Amsterdam, Amsterdam, The Netherlands; and ${ }^{6}$ Department of Microbiology and the Cancer Center, University of Virginia Health System, Charlottesville, Virginia
}

Note: Supplementary data for this article are available at Cancer Research Online (http://cancerres.aacrjournals.org/).

Corresponding Author: Bekir Cinar, Departments of Medicine-Hematology/Oncology and Biological Sciences, Samuel Oschin Comprehensive Cancer Institute, Cedars-Sinai Medical Center, University of California, 8750 Beverly Blvd., Atrium 103, Los Angeles, CA 90048. Phone: 310-4238658; Fax: 310-423-8543. E-mail: bekir.cinar@cshs.org

doi: 10.1158/0008-5472.CAN-10-4532

C2011 American Association for Cancer Research. caspase- 3 cleavage in response to a wide range of cell death stimuli (4).

In addition to their proapoptotic function, MST1 and its closest paralog MST2 have been shown to play an important role in mammalian development $(5,6)$, cell-cycle progression and tumorigenesis (7-10). For example, hpo deficiency in the developing Drosophila eye results in massive overgrowth due to an accelerated rate of proliferation and failure of developmental apoptosis (11-13). Likewise, MST1 or MST2 deficiency in mice is embryonically lethal (5). Loss or reduction of MST1 and MST2 expression has also been correlated with poor cancer prognosis (14). Recent genetic studies have indicated that liver-specific deletion of MST1 and MST2 in mice resulted in liver enlargement, cancer, and resistance to TNF- $\alpha$ induced apoptosis $(7,9,10)$. Previous studies suggest that cross talk between androgen receptor (AR) and MST1 signaling may have important biological consequences in prostate cancer (PCa; refs. 15, 16). Therefore, we wanted to investigate whether MST1 functionally intersects with AR signaling and regulates the growth of PCa cells.

Here, we show that MST1 is a novel negative regulator of AR signaling. Our data suggest that MST1 attenuates AR activity via a mechanism that involves protein complex formation between AR and MST1 in a manner that is independent of its kinase activity. Furthermore, we provide evidence that enforced MST1 expression suppressed PCa cell growth, sensitized androgen-independent C4-2 cells to phosphatidylinositol 3-kinase (PI3K) inhibition, and attenuated tumor growth in vivo. These findings suggest that loss of MST1 signaling may promote hyperactivation of AR and may be associated with the emergence of the castration-resistant phenotype. 


\section{Materials and Methods}

\section{Plasmid constructions, antibodies, and reagents}

The construction of HA- or Myc-tagged MST1-wt and Myc-MST1-N and Myc-MST1-C forms was described previously (15). For the construction of Doxycycline(Dox)-inducible HA-MST1 plasmid, PCR-amplified HA-tagged MST1-wt cDNA was inserted into BamHI and MluI enzyme sites in the pRetro-X-Pur vector (Clontech Laboratories, Inc.), designated as pRXTP-HA-MST1. The construction of glutathione S-transferase (GST)-AR DBD/HR (AR DNA binding domain and hinge region) was described previously (17). MST1 and AR point mutations were generated by using the QuickChange site-directed mutagenesis kit (Stratagene). The orientation and fidelity of all constructs were confirmed by DNA sequencing. Note that names of antibodies and reagents and their sources are provided in the Supplementary Information section.

\section{Cell transfections, reporter assays, and immunocytochemistry}

LNCaP and C4-2 were cultured in RPMI 1640 medium and HEK 293T, and COS-7 cells were cultured in DMEM at $37^{\circ} \mathrm{C}$ in $5 \% \mathrm{CO}_{2}$ incubator. Media were supplemented with $10 \%$ FBS and $1 \%$ penicillin/streptomycin. RNAi (siRNA) transfections with Dharma FECT 2 and plasmids transfections with Lipofectamine 2000 were carried out according to the manufacturer's instructions (Invitrogen). Luciferase reporter gene activities were measured by using the Luciferase Assay System from Promega and a BMG Labtech microplate reader. Immunocytochemistry (IHC) was done as described previously (15). Relative luciferase units were normalized to total protein and the result presented as luciferase (Luc) activity. Cells were imaged at $63 \times$ with a Plan-Apochromat oil immersion lens on an Axioplan 2 Apotome epifluorescence microscope (Zeiss). IHC was done by using reagents from DAKO and images were acquired at $20 \times$ with Nikon Imaging System.

\section{Establishment of TetON-inducible cells}

Retroviruses carrying Tet-repressor or HA-MST1 expression constructs were produced in HEK 293T cells expressing viral packaging proteins and then viral particles were concentrated by using $P E G$-it solution. LNCaP parental cells or its castration-resistant subline, C4-2, were first infected with retrovirus encoding pRetroX-TetON advanced plasmid, followed by selection with Geneticin $(\mathrm{G} 418,500 \mu \mathrm{g} / \mathrm{mL})$ to generate the TetON cells. The LNCaP/ or $\mathrm{C} 4-2 / \mathrm{TetON}$ cells were then infected with retrovirus encoding pRXTP-HA-MST1 vector, followed by Puromycin selection $(3 \mu \mathrm{g} / \mathrm{mL})$ to generate TetON inducible MST1 expressing cells. The inducible system allows fine control of MST1 expression. All protocols and procedures were done according to the manufacturer's instructions (Clontech Laboratories, Inc.).

\section{Protein analyses}

Cell lysis was conducted in buffer consisting of $20 \mathrm{mmol} / \mathrm{L}$ HEPES, pH 7.4, $150 \mathrm{mmol} / \mathrm{L} \mathrm{NaCl}, 0.5 \% \mathrm{NP}-40,1 \mathrm{mmol} / \mathrm{L}$
EDTA, protease inhibitors, and phosphatase inhibitor. For immunoprecipitation, cleared lysates were incubated with antibody overnight at $4^{\circ} \mathrm{C}$. Antibody-antigen complexes were collected on protein A- or G-sepharose and washed 3 times with cell lysis buffer. Immunoprecipitates were resolved by SDS-PAGE. PBST (0.1\% Tween-20) containing 5\% (w/v) skim milk powder or PBST containing $5 \%$ immunoglobulin G (IgG)free bovine serum albumin (Sigma) was used in membrane blocking and antibody dilutions. Signals were visualized by chemiluminescence. GST-AR DBD/HR and its mutant forms were expressed in bacteria with IPTG (Isopropyl $\beta$-D-1-thiogalactopyranosid) induction and protein purification was done with GST-sepharose by using a standard protocol. Cytoplasmic and nuclear fractions were prepared as described (18).

\section{Chromatin immunoprecipitation assays}

Chromatin immunoprecipitation (ChIP) was done as described previously (19). Briefly, LNCaP or C4-2/HA-MST1 cells grown in serum-starved conditions were treated with R1881 (1 nmol/L) or EtOH (vehicle) overnight. Dox $(-/+)$ used to induce MST1 expression in C4-2/HA-MST1 cells. DNA enriched with anti-MST1, anti-AR, or anti-Pol II antibody were quantified by semiquantitative PCR by using primer sets surrounding the AREIII region within the androgen-responsive element enhancer (ARE) core (AREc) or AREI of the prostate-specific antigen (PSA) promoter (18).

\section{Kinase, cell death, and cell proliferation assays}

For in vitro kinase assay, the recombinant, preactivated MST1 protein kinase was incubated with purified GST-AR DBD/HR fusion protein and $10 \mu \mathrm{Ci}{ }^{32} \mathrm{P}-\gamma$-ATP or $100 \mu \mathrm{mol} / \mathrm{l}$ unlabeled-ATP. The reaction mixture was resolved on SDSPAGE and autoradiographed. Cell Death ELISA and BrdU incorporation assays were conducted to assess cell death and cell proliferation, respectively, according to the manufacturer's instructions (Roche Molecular Diagnostics).

\section{Animal studies}

C4-2/Vector or C4-2/HA-MST1 cells mixed with Matrigel (1to-1 ratio) were inoculated s.c. in athymic nude mice (CD-1 $\mathrm{nu} / \mathrm{nu}$; Charles River Laboratories). A total of $1 \times 10^{6}$ cells $/ 100$ $\mu \mathrm{L}$ were used per injection per site (right and left flanks). Mice were treated with Dox $(0.5 \mathrm{mg} / \mathrm{mL})$ in drinking water to induce MST1 expression for 12 weeks. Institutional Animal Care and Use Committee policies and guidelines were strictly applied. Tumor volumes were assessed by caliper according to procedures described previously (20). Mice were sacrificed and evaluated for tumor growth anatomically and tumor tissues extracted from mice were fixed in $10 \%$ formaldehyde for the construction of histologic sections or "snap" frozen at $-80^{\circ} \mathrm{C}$. The expression of MST1 was verified in histologic sections by IHC by using anti-HA antibody.

\section{Statistics}

Data are represented as mean \pm SEM. Student $t$ test (2tailed) was used between the data pairs in which it is appropriate. A $P$ value 0.05 or less was considered significant. 
Figure 1. MST1 forms a protein complex with $A R$ and antagonizes AR activity. A, co-IP of ectopically expressed human MST1 and endogenous AR in LNCaP (top) or exogenously expressed human AR in HEK 293 (bottom) cells. CoIP and Western blots (WB) were done with corresponding antibodies. B, AR-responsive PSA promoter reporter (p61-Luc) activity in LNCaP under condition in which MST1 was knocked down (top) or enforced (bottom). An unpaired $t$ test was conducted to analyze for differences between treatments. *, $P \leq 0.02$. C, ARresponsive GRE4-Luc in $\mathrm{LNCaP}$ (top) and p61-Luc activity in COS7 cells (bottom). Serum-starved cells were treated with $(+)$ or without $1 \mathrm{nmol} / \mathrm{L}$ R1881, androgen analog, ${ }^{*}, P \leq 0.02$. All assays were conducted at 36 hour. Relative luciferase units presented as luciferase (Luc) activity after normalization with total protein. Data are representative of multiple experiments.

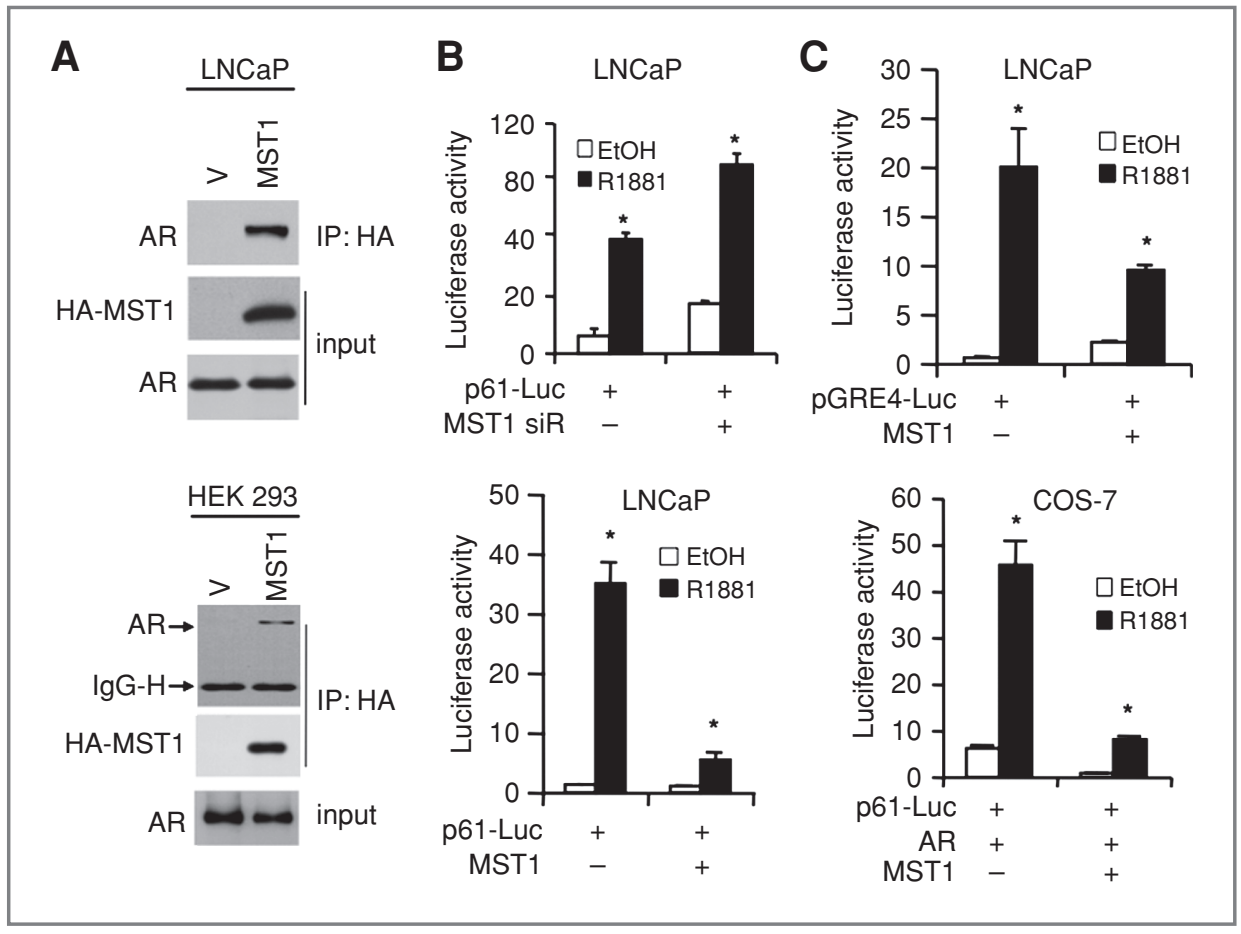

\section{Results}

\section{MST1 binds and attenuates AR activity}

To determine whether the MST1 kinase biochemically and functionally intersects with AR signaling, we employed cellular and biochemical approaches by using prostate and non-PCa cell lines that express either native, stable or transient AR. Coimmunoprecipitation (Co-IP) and Western blot experiments showed that MST1 interacts with endogenous AR in LNCaP cells (Fig. 1A, top panel) and ectopically expressed human AR in HEK 293 cells (Fig. 1A, bottom panel). Similarly, endogenous MST1 could also be found in the AR protein complex from PC3-hAR cells that were engineered to express near-physiologic levels of stable His-tagged human AR (18), as shown by Ni-NTA precipitation and Western blot experiments (Supplementary Fig. S1A and B).

To test whether the binding of MST1 has an impact on AR activity, we conducted AR-dependent promoter reporter assays $(18,21)$ by using knockdown and induction approaches. MST1 knockdown by a gene-specific siRNA (15) resulted in the upregulation of basal and androgen-stimulated AR-responsive PSA promoter reporter activation, by at least 2-fold in comparison with siRNA control (Fig. 1B, top panel). Enforced MST1 expression attenuated endogenous AR activity in LNCaP cells, as shown by the PSA promoter reporter (p61Luc) assay (Fig. 1B, bottom panel). Enforced MST1 expression also attenuated AR-dependent GRE4-driven simple promoter reporter (pGRE4-TATA-Luc) activation in LNCaP (Fig. 1C, top panel) and p61-Luc promoter reporter activation in COS-7 (Fig. 1C, bottom panel) cells, in which AR expression was also enforced. Collectively, these observations indicate that MST1 is a binding partner and a physiologic negative regulator of AR signaling.

\section{The full-length MST1 is a dominant AR suppressor}

To map the AR binding domain on MST1, the full-length AR was coexpressed with vector, MST1-wt or MST1-N (residues 1-330) or MST1-C (residues 331-487) truncation mutants in COS-7 cells, followed by co-IP and Western blot analysis. The results showed that the full-length MST1 (MST1-wt) and MST1-N strongly interacted, whereas MST1-C displayed weak interaction with exogenous AR (Fig. 2A). The inhibition of AR activity by MST1-wt, MST1-N, or MST1-C coincided with the binding data, as revealed by the PSA promoter reporter assay (Fig. 2B).

To determine which MST1 form (MST1-wt or the cleaved MST1-N) functions as a dominant AR inhibitor, we generated caspase-resistant single $[\mathrm{D} 326 \mathrm{~N}=\mathrm{D} / \mathrm{N}$, Asp (D) $\rightarrow$ Asn $(\mathrm{N})$ or $\mathrm{D} 349 \mathrm{E}=\mathrm{D} / \mathrm{E}, \mathrm{Asp} \rightarrow \mathrm{Glu}(\mathrm{E})]$ and double (D326N/ $\mathrm{D} 349 \mathrm{E}=\mathrm{DD} / \mathrm{NE}$ ) MST1 mutants and assessed their effects on PSA protein levels or luciferase reporter activity mediated by AR. The results show (Fig. $2 \mathrm{C}$ and $\mathrm{D}$ ) that the expression of each MST1 mutant is capable of inhibiting endogenous PSA protein levels and PSA promoter reporter activation induced by androgen, similar to the levels seen with MST1wt. Neither the expression of MST1-wt nor these MST1 mutants affected AR protein levels, unless the cells were stimulated by androgen (Fig. 2C, AR blot). A similar level of AR-dependent PSA promoter reporter inactivation by MST1 was also obtained, even in the presence of increasing doses of a specific caspase inhibitor, Ac-DEVD-CHO (D-CHO; Supplementary Fig. S2A). 


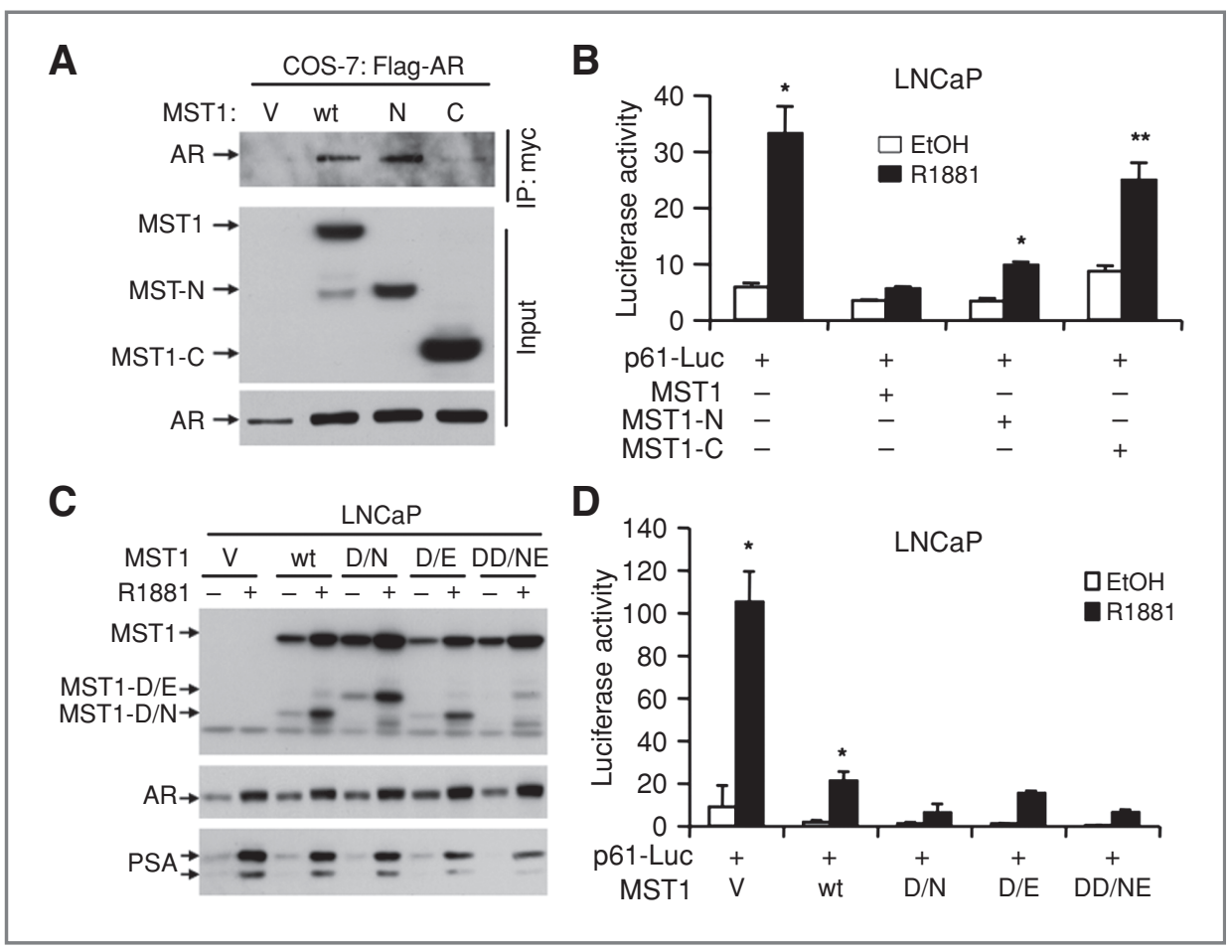

Figure 2. Caspase cleavagedeficient MST1 is a potent AR inhibitor. A, AR binding domains on MST1. AR with vector $(\mathrm{V}$, MST1-wt (wild type) or MST1 domains (MST1-N or MST1-C) was transiently coexpressed in COS-7 cells. Co-IP and WB were done with antibodies to corresponding proteins. B, PSA promoter reporter activity in LNCaP cells with MST1-wt or MST1 truncation mutant in A, ${ }^{*}, P \leq 0.01 ;{ }^{* *}, P \leq 0.08$. C, Western blots of MST1, AR or PSA protein in LNCaP cells transfected with Mock, MST1-wt, MST1D326N (D/N), MST1-D349E (D/E), or MST1-D326N/D349E (DD/NE), followed by R1881 (+) or vehicle $(-)$ treatment in serum-starved conditions. D, PSA promoter reporter activity with caspaseresistant MST1 constructs under the same conditions as described in $\mathrm{C},{ }^{*}, P \leq 0.01$. Luciferase activity was determined at 36 hour. Data are representative of multiple experiments.

We then examined protein complexes between $\mathrm{AR}$ and the caspase-deficient MST1-DD/NE mutant. AR was coexpressed with vector, MST1-wt, or MST1-DD/NE mutant constructs in HEK 293 cells. As shown by co-IP/Western blot experiments, the MST1-DD/NE mutant maintained interaction with AR and the levels of interaction between AR and MST1-DD/NE were similar or even greater than that observed with MST1-wt (Supplementary Fig. S2B). These observations indicate that the caspase-deficient MST1 is a potent AR inhibitor.

\section{MST1 attenuates AR activity in a Ser650 phosphorylation-independent manner}

MST1 is a stress-induced kinase (2), and other stressinduced kinases such as c-Jun-N-Terminal Protein Kinase 1 (JNK1) or p38 mitogen-activated protein (MAP) kinase have been proposed to physically interact with and antagonize AR transcriptional activity by phosphorylating AR at Ser650 (17). To determine whether purified MST1 can also physically interact with and phosphorylate AR at this site, we conducted the GST-pulldown and an in vitro kinase assay by using recombinant, preactivated MST1, and purified GST-AR$\mathrm{DBD} / \mathrm{HR}$ as a substrate. The results of these experiments revealed that preactivated, recombinant MST1 physically interacted with (Fig. 3A) and specifically phosphorylated the purified GST-AR-DBD/HR fragment in vitro (Fig. 3B, left panel). Both binding and phosphorylation events occurred in a dose-dependent manner (Supplementary Fig. S3A and B, respectively). Using a site-specific phospho-AR antibody, we were able to identify the Ser650 residue as a target of the MST1 kinase activity, as revealed by nonradioactive in vitro kinase assay and Western blot experiments (Fig. 3B, right panel).
To determine whether Ser650 phosphorylation has a role in the attenuation of AR activity by MST1, we generated phosphorylation-inactivating $\quad(\mathrm{Ser} \rightarrow \mathrm{Ala})$ or phosphomimetic (Ser $\rightarrow \mathrm{Glu}$ ) mutations and assessed their impact on MST1mediated inhibition of AR activity. The data in Figure 3C show that enforced-MST1 expression is capable of inhibiting AR transcriptional activity regardless of the presence of phosphorylation-inactivating Ser650A or phosphomimetic Ser650D AR mutations. Similarly, other site-specific phosphorylation-inactivating mutations did not significantly affect AR inhibition by MST1 induction (Supplementary Fig. S3C). In addition, phosphorylation-inactivating or phosphomimetic mutations did not alter ligand-dependent nuclear localization of $\mathrm{AR}$ in COS-7 cells expressing exogenous AR (Fig. 3D). Furthermore, co-IP/Western blot analyses showed that neither type of mutation affected complex formation between the two proteins (Supplementary Fig. S3D). In addition, with the exception of the Ser308A mutation, these known phospho-site mutations do not significantly alter androgen-induced AR transcriptional activity in COS-7 cells (Supplementary Fig. S4A). These findings indicate that the phosphorylation of Ser650 by MST1 is not involved in the mediation of AR inhibition by MST1.

\section{MST1 kinase activity is not required for the inhibition of AR activity}

To determine whether MST1 kinase activity has an effect on the inhibition of AR transcriptional activity, we generated kinase-deficient MST1 mutants (MST1-K59R in the ATP binding pocket and MST1-T183A in the activation loop). Consistent with published data (3), neither of these MST1 mutants was able to induce apoptosis in COS-7 or in LNCaP cells, compared 


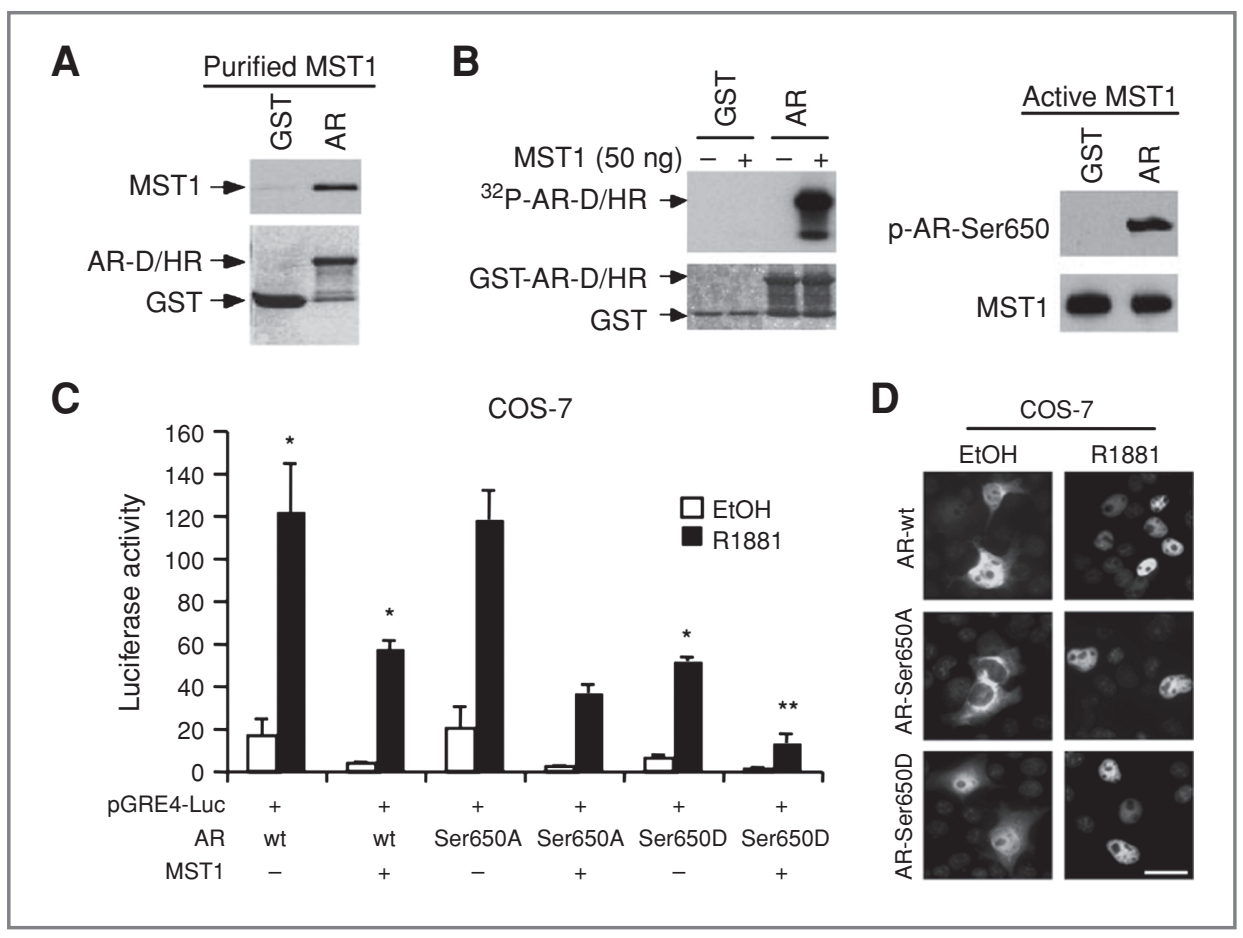

Figure 3. Purified recombinant MST1 binds and phosphorylates GST-AR-D/HR fragment at Ser650. A and B, GST-pull down in A and in vitro kinase assay with bacterially expressed GST-AR-D/HR and recombinant, preactivated MST1 protein kinase in B, left. Right, WB probed with antibody to MST1 or to phospho-Ser650 AR in B. AR-D/HR: AR-DNA binding domain/hinge region. C, pGRE4-Luc promoter luciferase activity in COS-7 cells. Cells were cotransfected with pGRE4-Luc and corresponding constructs and treated with R1881 (+) or vehicle $(-)$ in serum-starved conditions, ${ }^{*}, * *, P \leq 0.01$. Luciferase reporter assay was conducted at 36 hour following androgen treatment. D, immunofluorescence staining of AR protein (FITC, cytoplasm with EtOH vehicle and nuclear with R1881) with anti-AR antibody and DAPI (nucleus) in COS-7 cells transfected to express transient AR and stimulated with R1881 ( + ) or vehicle (-) for 6 hour in serum-starved conditions. The size bar represents 10 microns. Data are representative of multiple experiments.

with MST1-wt (Fig. 4A). However, both mutants were capable of inhibiting AR-driven PSA promoter activation, similar to the levels observed with MST1-wt in LNCaP (Fig. 4B) and in its castration-resistant C4-2 subline (Supplementary Fig. S4B). We then examined the protein complexes between AR and these kinase-inactivated MST1 mutants. Mock, MST1-wt, MST1K59R, or MST1-T183A constructs were coexpressed with AR in COS-7 cells and co-IP experiments were done by using total cell lysates. Western blot analysis with anti-AR antibody revealed that the kinase-inactivating mutation had no affect on the formation of the AR and MST1 complex (Fig. 4C). We also conducted the promoter reporter assay with MST2 and showed that transient expression of this close structural relative to MST1 also antagonized the AR transactivation function in LNCaP cells independently of its kinase activity (Fig. 4D). These results suggest that both kinases perform a similar inhibitory role with respect to the AR, and that neither the kinase activity nor the proapoptotic function of MST1 or MST2 is involved in the inhibition of AR activity.

\section{MST1 suppresses AR activity by intersecting with AKT1 signaling and antagonizing formation of AR-chromatin complexes}

An implication from the above findings is that additional mechanisms may be involved in MST1-mediated AR inhibi- tion. MST1 was reported to inhibit AKT signaling (15), which is known to functionally intersect with $(16,22)$ and promote ARdriven PSA promoter activation (23). To test whether MST1 induction could suppress AR activation mediated by AKT1 signaling, we conducted promoter reporter assays and showed that enforced MST1 expression antagonized AKT1 mediated androgen-dependent and -independent AR activation (Fig. 5A, left panel). Co-IP experiments further revealed that MST1, AR, and AKT1 form a tri-partite complex in vivo (Fig. 5A, right panel), indicating that MST1 also intersects with AKT signaling to attenuate AR activity.

Given that MST1 forms protein complexes with AR, we showed by using co-IP experiments that endogenous AR and MST1 form a complex preferentially in cell nuclei (Fig. 5B). These observations led us to investigate whether MST1 localizes within AR-transcriptional complexes. ChIP experiments showed that endogenous MST1 interacts with the PSA promoter region, which also binds $\mathrm{AR}$ in both serum- and androgen-free conditions and can be regulated by androgen in LNCaP cells (Fig. 5C). This segment of the PSA promoter, referred to as the ARE core, consists of multiple AREs and plays a prominent role in AR-driven PSA promoter regulation $(18,21)$. Additional data suggest that MST1 also binds ARE-I and the TATA region in the PSA promoter, which can also be regulated by androgen (Fig. 5D). Taken together, these 
A

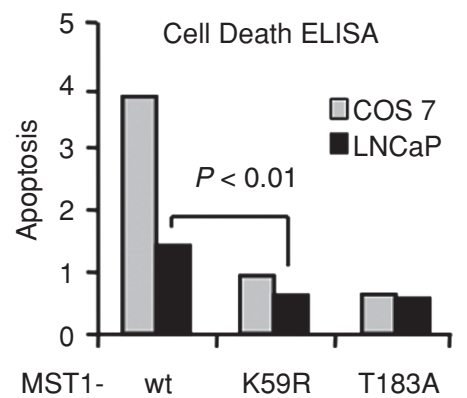

C

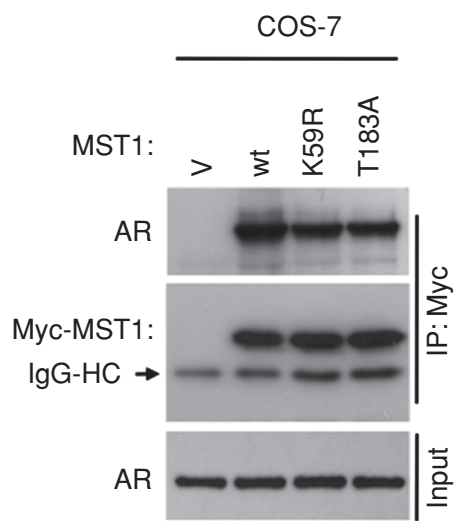

B

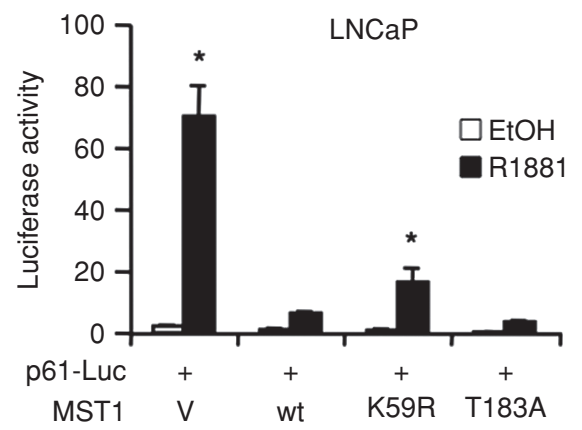

D

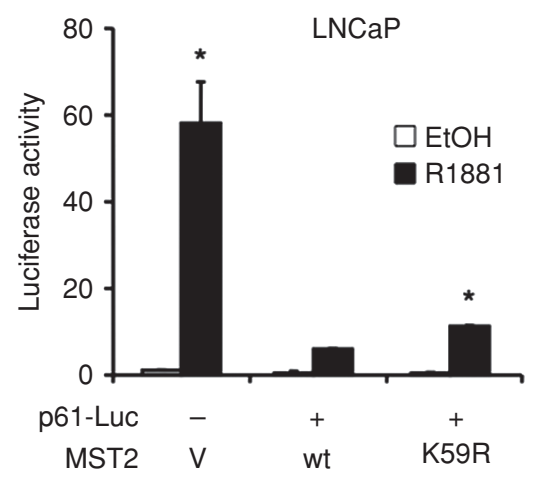

Figure 4. The kinase and apoptotic functions of MST1 are not involved in AR inhibition. A, apoptosis in LNCaP or in COS-7 cells transfected with MST1-wt or kinase deficient-MST1 mutant construct (MST1-K59R or MST1T183A). Cell apoptosis was determined by using Cell Death ELISA. B, PSA promoter reporter activity in LNCaP cells with kinase intact or kinase-deficient MST1 as in $\mathrm{A}$, followed by incubation with (+) or without (-) R1881. C, MST1 and AR protein complex in COS-7 cells expressing AR along with vector, MST-wt, or kinasedeficient MST1 mutant. Co-IP and Western blots were conducted in total cell lysates with antibodies to corresponding proteins. D, MST2regulated PSA promoter reporter activity in LNCaP cells, * $P \leq 0.01$. p61-Luc (+) was cotransfected with MST2-wt or with kinasedeficient MST2 mutant (MST2K56R), ${ }^{*}, P \leq 0.01$. All assays were conducted at 36 hour. HC: IgGheavy chain. Data are representative of multiple experiments. findings suggest that MST1 reduces AR-chromatin complex formation to attenuate androgenic signals.

\section{MST1 suppresses PCa cell growth in vitro and tumor growth in vivo}

To address the impact of MST1 on cell growth, we established stable MST1 expressing LNCaP/HA-MST1 or C4-2/HA-MST1 cells by using a retroviral inducible system. LNCaP/HA-MST1 or C4-2/HA-MST1 cells were exposed to increasing doses of Dox. Dose-dependent induction of MST1 expression reduced the growth of LNCaP; however, castrationresistant LNCaP subline, C4-2, displayed resistance to the growth suppressive effects of enforced MST1 (Fig. 6A and B). The observed growth reduction originated from MST1 induction because the administration of the highest dose of Dox $(4 \mu \mathrm{g} / \mathrm{mL})$, which reduced the growth of $\mathrm{LNCaP} / \mathrm{HA}$ MST1 the most, had no effect on the growth of LNCaP/Vector cells (Supplementary Fig. S5A). In addition, enforced MST1 expression in C4-2/HA-MST1 cells dramatically reduced the number and size of C4-2 colonies grown in soft agar (Fig. 6C) and sensitized C4-2 cells to growth suppression induced by PI3K inhibitor LY294002 (Fig. 6D). C4-2 cells are relatively resistant to PI3K inhibitors (24) and less sensitive to the effects of MST1 compared with parental LNCaP cells (Fig. 6A). As expected, induction of MST1 prevented the growth of LNCaP cells regardless of the presence of PI3K inhibitor (Supplementary Fig. S5B).

To assess the distribution of MST1, we analyzed levels of exogenous MST1 protein in cytoplasmic and nuclear fractions obtained from C4-2/HA-MST1 cells. The result revealed that although the majority of exogenous HA-MST1-wt localized in the cytoplasm, significant levels of exogenous HA-MST1-wt protein were also found in the nucleus (Fig. 7A, left panel). Immunofluorescence experiments confirmed these results (Supplementary Fig. S5C). Stable MST1 expression was capable of inhibiting AR-driven PSA promoter activation in C4-2/ HA-MST1 cells (Fig. 7A, right panel). ChIP experiments by using lysates from C4-2/HA-MST1 cells further showed that loading of MST1 onto the ARE core region of the PSA promoter diminished the formation of AR-chromatin complexes (Fig. 7B).

To determine the physiologic relevance of our in vitro findings, we carried out xenograft experiments. C4-2/HAMST1 or C4-2/Vector cells were inoculated s.c. into immunodeficient male mice and the animals were then treated with Dox in the drinking water. Immunohistochemical analyses of the resultant tumors verified the expression of HA-MST1 in histologic sections from C4-2/HA-MST1 or C4-2/Vector tumor xenografts (Fig. 7C, micrograph). Consistent with observations in vitro, enforced MST1 expression suppressed the growth by 


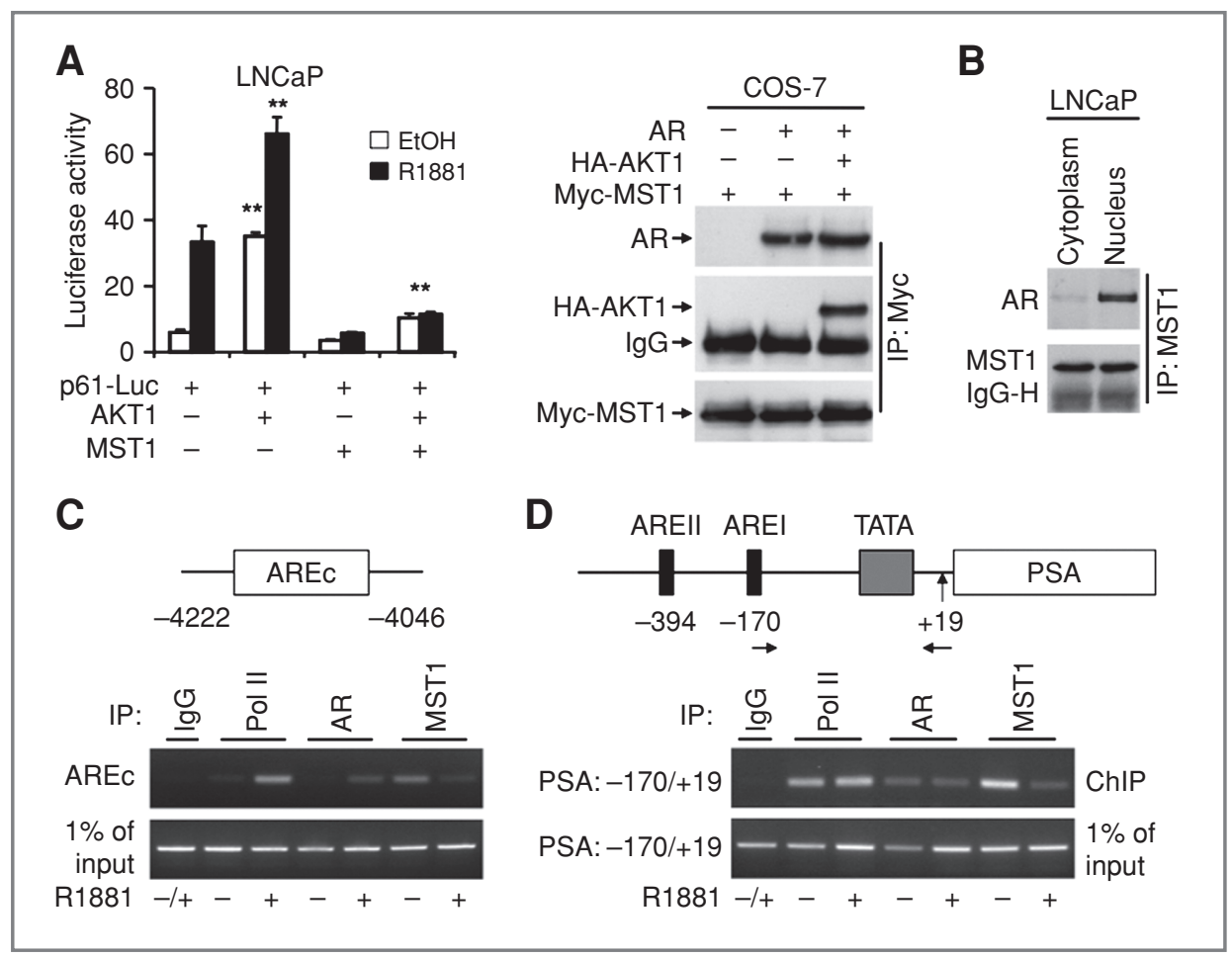

Figure 5. MST1 antagonizes AKT-mediated AR activation and localizes to AR-chromatin complexes. A, graph: PSA promoter reporter activity modulated with MST1 and AKT signaling, *, **, $P \leq 0.01$. LNCaP cells were transiently cotransfected with p61-Luc promoter reporter and AKT1 or MST1 alone or together with AKT1 and MST1, followed by androgen or vehicle treatment in serum-starved conditions. Blots: A tri-partite protein complex between AR, MST1, and AKT1 in COS-7 cells expressing transient MST1 along with AR or AR and AKT1. All assays were conducted at 36 hour. HC: IgG-heavy chain. B, co-IP and WB analysis of protein complexes between endogenous AR and MST1 in LNCaP cells grown in basal condition. C and D, ChIP assay in lysates of LNCaP cells. Protein-DNA complexes were precipitated with IgG (negative control), Pol II (positive control), AR, or MST1 antibody in total cell lysates. DNA interaction was assessed by semiquantitative PCR by using primers surrounding AREIII within the AREc and surrounding AREI and TATA region of the PSA promoter. Diagram showing AREc and proximal PSA promoter region where multiple AREs and TATA box, AREI, and AREll reside, respectively. Primer pair against PSA gene was used in input control. Data are representative of multiple experiments.

35 -fold and the tumorigenic rates by 4-fold of C4-2/HA-MST1 cells compared with C4-2/Vector counterparts (Fig. 7C, graph and tumor mass image).

\section{Discussion}

In this study, we show that the serine-threonine kinase MST1 is a physiologic negative regulator of AR signaling. We provide evidence that MST1 forms protein complexes in vitro and in vivo with $\mathrm{AR}$ and antagonizes AR activity in multiple cell backgrounds. Although both the full-length MST1 and the cleaved MST1-N forms bound and inhibited AR activity, caspase-resistant MST1 was the most potent AR inhibitor. We found that the kinase activity of MST1 was not required for the attenuation of AR activity. Similarly, the proapoptotic function of MST1 is not involved in AR inhibition because kinase deficient MST1, which failed to induce cell death, was capable of interacting with $\mathrm{AR}$ and inhibiting $\mathrm{AR}$ transcriptional activity. Furthermore, promoter reporter and ChIP experiments revealed that enforced MST1 antagonized AKTmediated AR activation and reduced binding of $A R$ to its cognate DNA binding site. On the basis of these observations, we propose that MST1 antagonizes AR-dependent gene expression by forming inhibitory protein and/or transcriptional complexes with AR, thereby suppressing prostate tumor growth.

Posttranslational modifications, such as phosphorylation (17), palmitoylation (25), ubiquitination (26), acetylation (27), or SUMOylation (28) play important roles in the regulation of AR activity. Several phosphorylation sites at serine residues and a tyrosine residue have been identified in AR $(29,30)$. These modifications negatively or positively regulate AR activity in a context-dependent manner (24), and their functional significance in PCa is beginning to emerge (31). For example, the phosphorylation of AR at Tyr-534 by cSrc was shown to enhance AR activation and AR-dependent gene expression, which was shown to be correlated with hormone-refractory $\mathrm{PCa}$ (30). On the other hand, phosphorylation of AR at Ser650 by JNK1 or p38 MAP kinase was shown to inhibit AR activity (17). Here, we showed that MST1 is an AR kinase and phosphorylates AR at Ser650 (Fig. 3C). However, phosphorylation of Ser650 by MST1 had no effect on the observed inhibition of AR-transcriptional activity. Nevertheless, the role of phospho-Ser650 on mechanisms of AR action deserves further investigation, 


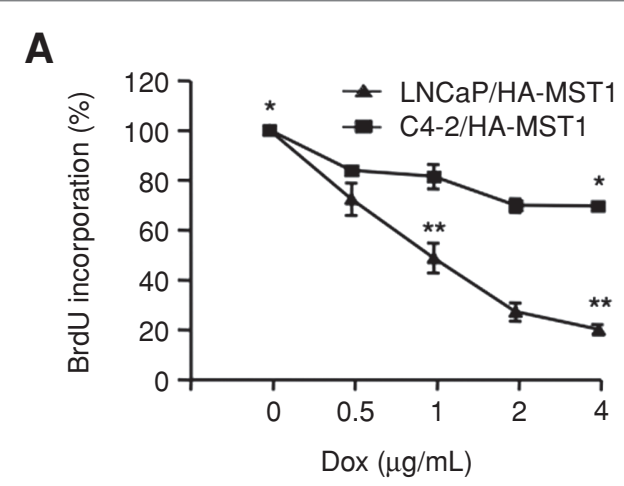

C

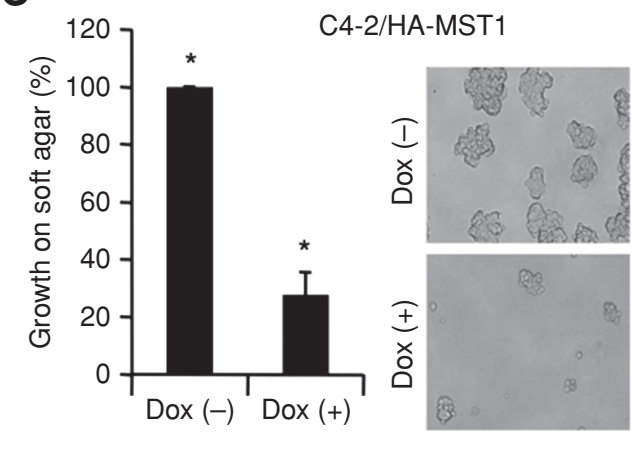

B

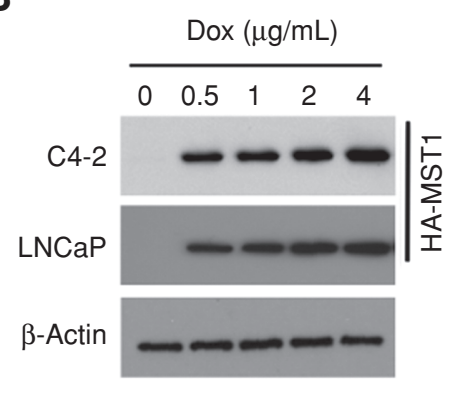

D

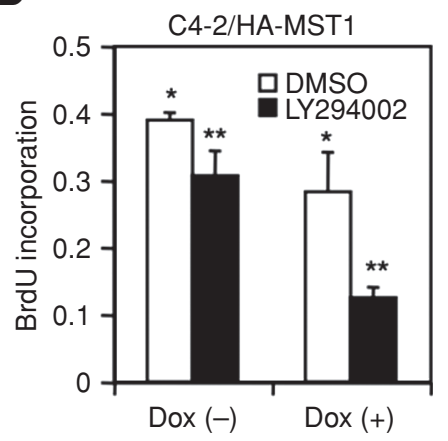

Figure 6. Enforced MST1 expression suppresses prostate tumor cell growth in monolayer culture and colony formation on soft agar. A, cell proliferation with BrdU incorporation assay, ${ }^{*}, P \leq 0.2 ;{ }^{* *}, P \leq 0.0009$. B, stable MST1 expression by Western blot. LNCaP/HA-MST1 and C4-2/HA-MST1 cells treated with increasing doses $(0,0.5,1,2$, and $4 \mu \mathrm{g} / \mathrm{mL})$ of Dox, respectively, in A and B. Western blots probed with anti-HA antibody or anti- $\beta$ - actin as a loading control. C, colony formation assay on soft agar. Equal numbers of C4-2/HA-MST1 cells were seeded on soft agar and grown for 14 days in the presence $(0.5 \mu \mathrm{g} / \mathrm{mL})$ and absence $(-)$ of Dox in regular growth conditions with the media was replaced every 3 days; ${ }^{*}, P \leq 0.004$. The graph represents the quantification of colonies and the micrograph represents colonies formed on soft agar. D, the growth of C4-2/HA-MST1 cells treated with LY294002 $(20 \mu \mathrm{mol} / \mathrm{L})$, a potent PI3K inhibitor or vehicle [dimethyl sulfoxide (DMSO)] in the presence $(+)$ and absence $(-)$ of $\mathrm{Dox},{ }^{*}, P \leq 0.08 ;{ }^{* *}, P \leq 0.007$.

All cell proliferation was determined at 24 hour. Data are representative of multiple experiments.

given that phosphorylation-inactivating Ser650A or phosphomimetic Ser650D AR mutations are able to alter the cellular distribution of $\mathrm{AR}$ in comparison to that observed with AR-wt under androgen-free conditions (Fig. 3D). Our data do not rule out the possibility that the existence of other potential MST1 phosphorylation sites might play a role in the inhibition of AR activity by MST1. In addition, the induction of MST1 was shown to activate JNK1 (32), which is known to inhibit AR (17). We did not observe JNK1 activation in response to MST1 induction in LNCaP, unless cells were treated with phorbol ester (Supplementary Fig. S2C). This indicates that MST1 attenuates AR signaling by a distinct mechanism apart from JNK or p38 MAP kinase activation.

In addition to posttranslational modifications, transcriptional coregulators (i.e., corepressors or coactivators) play an essential role in the modulation of AR activity, and their altered expression has been shown in prostate tumor progression (33). For example, the recruitment of nuclear corepressor (N-CoR) (34) or silencing mediator for thyroid and retinoid receptors (35) into the AR transcriptional complex was shown to antagonize AR activity by a mechanism involving protein-protein interaction. Similarly, displa- cement of coactivators such as p300 from the holo-AR transcriptional complex, or recruitment to the complex of histone deacetylase, which modifies chromatin structure to a transcriptionally inactive form (36), have also been shown to attenuate AR activity (35). Given that MST1 attenuates AR activity by forming protein complexes, the localization of MST1 into the holo-AR transcriptional complex could also attenuate the AR. Our data in Figures 5C, D and 7B support this conclusion; however, comprehensive studies are needed to elucidate precisely how MST1 alters AR-chromatin complexes.

MST1 and its downstream effectors, such as WW45 or LATS1/2, have been implicated in cancer, including PCa (37). For example, the liver specific knockout of MST1/2 expression in mice has been associated with hepatocellular carcinoma, which has been linked to the activation of YAP (7, 9, 10), and YAP is normally attenuated by the MST-LATS signaling network (10). The loss or reduced expression of LATS2 was reported in PCa and this was shown to be associated with hyperactivation of $\mathrm{AR}$ and upregulation of AR-dependent gene expression (38), with protein products known to promote PCa cell survival and inhibit apoptosis. In 


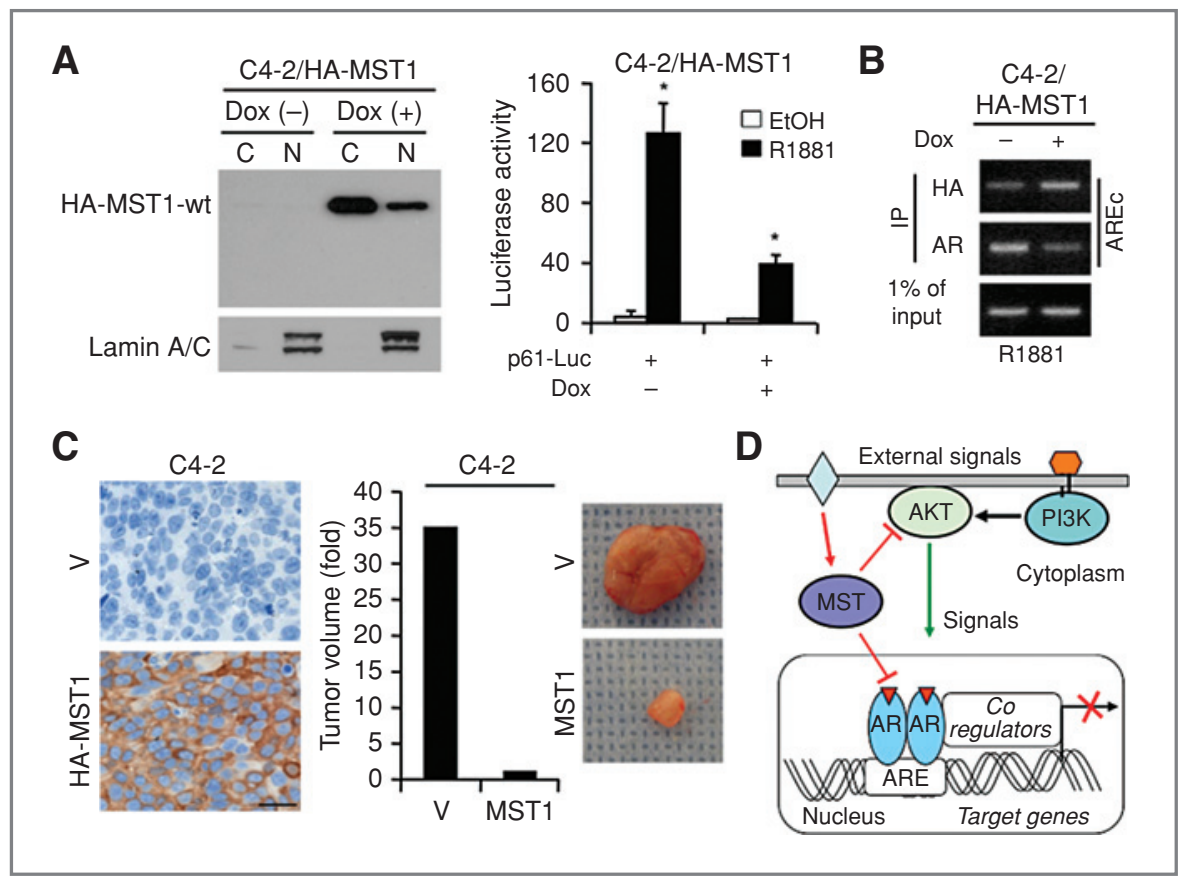

Figure 7. Enforced MST1 expression suppresses PCa xenografts. A, left, WB analysis of ectopically expressed HA-MST1 in cytoplasmic and nuclear cell fractions obtained from C4-2/HA-MST1 cells treated with Dox (-) or Dox (+). HA-MST1 was probed with anti-HA antibody. Lamin A/C was used as a nuclear extraction control. Right, PSA promoter reporter $(+)$ activity in C4-2/HA-MST1 cell treated with (+) or without (-) Dox and vehicle (EtOH) or androgen (R188) at 24 hour following treatment, ${ }^{*}, P \leq 0.01$. B, ChIP assay in lysates from C4-2/HA-MST1 cells, respectively. Protein-DNA complexes were precipitated with HA-tag antibody. DNA interaction was assessed by semiquantitative PCR by using primers surrounding ARElll within the AREc region of the PSA promoter. Primer pair against PSA gene was used in input control. C, left, immunohistochemical staining of HA-MST1 with anti-HA antibody in histologic sections from tissue xenografts of C4-2Nector or C4-2/HA-MST1 tumor. The size bar in C, left represents 100 microns. Right, the quantification of s.c. tumor growth in mice. Ten mice per group were used. Eight of 10 mice for C4-2/Nector or 2 of 10 mice for C4-2/HA-MST1 were developed tumors 12 weeks after s.c. inoculation of the cell. Data are representative of multiple experiments. D, diagram showing the points at which MST1 negatively regulates AR-mediated gene expression.

addition, mice lacking WW45 expression displayed hyperplasia in several organ sites (39). Here, we found that the induction of MST1 expression is sufficient to antagonize AR-driven gene expression and suppress $\mathrm{PCa}$ cell growth. Moreover, we found that the growth suppressive effects of MST1 significantly declined in castration-resistant C4-2 cells in comparison with the effects of MST1 in castration-sensitive LNCaP parental cells, though both cell models expressed similar levels of MST1 protein. MST1 was identified as a negative regulatory component of PI3K-AKT signaling, and reduced MST1 expression was shown to correlate with PCa progression to the hormone-refractory metastatic state, which coincides with AKT activation (15). Our data are consistent with this observation and indicate that the induction of MST1 expression sensitized C4-2 cells to growth suppression induced by PI3K inhibition. Our findings raise the possibility that deregulated-MST function may be associated with the emergence of the castration-resistant disease phenotype. The use of MST1/2 knockout mouse models will address the hypothesis of whether one or both of these kinases have a direct role in prostate carcinogenesis and emergence of castration-resistance.

PCa is the most commonly diagnosed cancer among men and the second leading cause of cancer death in Western countires $(40,41)$. Evidence indicates that cooperative $A R$ and PI3K/AKT-mTOR pathway signaling is critical to human prostate tumor development and progression to the metastatic phenotype (42-44). On the basis of published studies $(15,16)$ and our present findings, we propose a model (Fig. 7D) in which MST1/2 attenuates AR-dependent gene expression by interacting with the AR, which may lead to the alterations of the AR protein and/or transcriptional complexes, as well as by targeting upstream of the AR signal. An important implication from these observations is that deregulation of MST1 may account for the upregulation of AR and AKT signaling regulating cell survival. Therefore, disruption of the AR-AKT oncogenic network by MST1/2 alone and/or in combination with chemotherapeutic agents that target $\mathrm{AR}$ and PI3K/AKT-mTOR may have important therapeutic implications.

\section{Disclosure of Potential Conflicts of Interest}

No potential conflicts of interest were disclosed.

\section{Acknowledgments}

We thank Leland Chung, Rosalyn Adam, and Haiyen Zhau for their critical review of the data during the course of the study. We also thank Paul Guthrie, Mustafa Kupeli, Xiaojian Yang, and Yueming Wang for their technical assistance and Gary Mawyer for editorial suggestions. 


\section{Grant Support}

This study was supported by the Edwin Beer Fellowship grant (R24D00) from the New York Academy of Medicine and the Garber Foundation Award from Cedars-Sinai Medical Center (B. Cinar), NIH grants R01 CA124706 (D. Gioeli), R01 CA143777 and R01 CA112303 (M.R. Freeman), and U.S. Department of Defense grant W81XWH-08-1-0150 (M.R. Freeman).

\section{References}

1. Creasy CL, Chernoff J. Cloning and characterization of a member of the MST subfamily of Ste20-like kinases. Gene 1995;167:303-6.

2. Ling P, Lu TJ, Yuan CJ, Lai MD. Biosignaling of mammalian Ste20related kinases. Cell Signal 2008;20:1237-47.

3. Ura S, Masuyama N, Graves JD, Gotoh Y. Caspase cleavage of MST1 promotes nuclear translocation and chromatin condensation. Proc Natl Acad Sci U S A 2001;98:10148-53.

4. Teraishi F, Guo W, Zhang L, Dong F, Davis JJ, Sasazuki T, et al. Activation of sterile20-like kinase 1 in proteasome inhibitor bortezomib-induced apoptosis in oncogenic K-ras-transformed cells. Cancer Res 2006;66:6072-9.

5. Oh S, Lee D, Kim T, Kim TS, On HJ, Hwang CY, et al. Crucial role for Mst1 and Mst2 kinases in early embryonic development of the mouse. Mol Cell Biol 2009;29:6309-20.

6. Wu S, Huang J, Dong J, Pan D. Hippo encodes a Ste-20 family protein kinase that restricts cell proliferation and promotes apoptosis in conjunction with salvador and warts. Cell 2003;114:445-56.

7. Lu L, Li Y, Kim SM, Bossuyt W, Liu P, Qiu Q, et al. Hippo signaling is a potent in vivo growth and tumor suppressor pathway in the mammalian liver. Proc Natl Acad Sci U S A 2010;107:1437-42.

8. Praskova M, Xia F, Avruch J. MOBKL1A/MOBKL1B phosphorylation by MST1 and MST2 inhibits cell proliferation. Curr Biol 2008;18: 311-21.

9. Song H, Mak KK, Topol L, Yun K, Hu J, Garrett L, et al. Mammalian Mst1 and Mst2 kinases play essential roles in organ size control and tumor suppression. Proc Natl Acad Sci U S A 2010;107:1431-6.

10. Zhou D, Conrad C, Xia F, Park JS, Payer B, Yin Y, et al. Mst1 and Mst2 maintain hepatocyte quiescence and suppress hepatocellular carcinoma development through inactivation of the Yap1 oncogene. Cancer Cell 2009;16:425-38.

11. Harvey KF, Pfleger CM, Hariharan IK. The Drosophila Mst ortholog, hippo, restricts growth and cell proliferation and promotes apoptosis. Cell 2003;114:457-67.

12. Jia J, Zhang W, Wang B, Trinko R, Jiang J. The Drosophila Ste20 family kinase dMST functions as a tumor suppressor by restricting cell proliferation and promoting apoptosis. Genes Dev 2003;17: 2514-9.

13. Udan RS, Kango-Singh M, Nolo R, Tao C, Halder G. Hippo promotes proliferation arrest and apoptosis in the Salvador/Warts pathway. Nat Cell Biol 2003;5:914-20.

14. Seidel C, Schagdarsurengin U, Blumke K, Wurl P, Pfeifer GP, Hauptmann S, et al. Frequent hypermethylation of MST1 and MST2 in soft tissue sarcoma. Mol Carcinog 2007;46:865-71.

15. Cinar B, Fang PK, Lutchman M, Di Vizio D, Adam RM, Pavlova N, et al. The pro-apoptotic kinase Mst1 and its caspase cleavage products are direct inhibitors of Akt1. EMBO J 2007;26:4523-34.

16. Cinar B, Mukhopadhyay NK, Meng G, Freeman MR. Phosphoinositide 3-kinase-independent non-genomic signals transit from the androgen receptor to Akt1 in membrane raft microdomains. J Biol Chem 2007;282:29584-93.

17. Gioeli D, Black BE, Gordon V, Spencer A, Kesler CT, Eblen ST, et al. Stress kinase signaling regulates androgen receptor phosphorylation, transcription, and localization. Mol Endocrinol 2006;20:503-15.

18. Cinar B, Yeung F, Konaka H, Mayo MW, Freeman MR, Zhau HE, et al. Identification of a negative regulatory cis-element in the enhancer core region of the prostate-specific antigen promoter: implications for intersection of androgen receptor and nuclear factor-kappaB signalling in prostate cancer cells. Biochem J 2004;379:421-31.

19. Mukhopadhyay NK, Cinar B, Mukhopadhyay L, Lutchman M, Ferdinand AS, Kim J, et al. The zinc finger protein ras-responsive element binding protein-1 is a coregulator of the androgen receptor: implica-
The costs of publication of this article were defrayed in part by the payment of page charges. This article must therefore be hereby marked advertisement in accordance with 18 U.S.C. Section 1734 solely to indicate this fact.

Received December 17, 2010; revised March 8, 2011; accepted April 1, 2011; published OnlineFirst April 21, 2011.

tions for the role of the Ras pathway in enhancing androgenic signaling in prostate cancer. Mol Endocrinol 2007;21:2056-70.

20. Cinar B, Koeneman KS, Edlund M, Prins GS, Zhau HE, Chung LW. Androgen receptor mediates the reduced tumor growth, enhanced androgen responsiveness, and selected target gene transactivation in a human prostate cancer cell line. Cancer Res 2001;61:7310-7.

21. Cleutjens KB, Van Der Korput HA, van Eekelen CC, van Rooij HC, Faber PW, Trapman J. An androgen response element in a far upstream enhancer region is essential for high, androgen-regulated activity of the prostate-specific antigen promoter. Mol Endocrinol 1997;11:148-61.

22. Sun M, Yang L, Feldman RI, Sun $X M$, Bhalla $K N$, Jove $R$, et al. Activation of phosphatidylinositol 3-kinase/Akt pathway by androgen through interaction of p85alpha, androgen receptor, and Src. J Biol Chem 2003;278:42992-3000.

23. Wang Y, Kreisberg JI, Ghosh PM. Cross-talk between the androgen receptor and the phosphatidylinositol 3-kinase/Akt pathway in prostate cancer. Curr Cancer Drug Targets 2007;7:591-604.

24. Lin J, Adam RM, Santiestevan E, Freeman MR. The phosphatidylinositol 3'-kinase pathway is a dominant growth factor-activated cell survival pathway in LNCaP human prostate carcinoma cells. Cancer Res 1999;59:2891-7.

25. Pedram A, Razandi M, Sainson RC, Kim JK, Hughes CC, Levin ER. A conserved mechanism for steroid receptor translocation to the plasma membrane. J Biol Chem 2007;282:22278-88.

26. Xu K, Shimelis H, Linn DE, Jiang R, Yang X, Sun F, et al. Regulation of androgen receptor transcriptional activity and specificity by RNF6induced ubiquitination. Cancer Cell 2009;15:270-82.

27. Fu M, Rao M, Wu K, Wang C, Zhang X, Hessien M, et al. The androgen receptor acetylation site regulates CAMP and AKT but not ERKinduced activity. J Biol Chem 2004;279:29436-49.

28. Nishida T, Yasuda H. PIAS1 and PIASxalpha function as SUMO-E3 ligases toward androgen receptor and repress androgen receptordependent transcription. J Biol Chem 2002;277:41311-7.

29. Gioeli D, Ficarro SB, Kwiek JJ, Aaronson D, Hancock M, Catling AD, et al. Androgen receptor phosphorylation. Regulation and identification of the phosphorylation sites. J Biol Chem 2002;277:29304-14.

30. Guo Z, Dai B, Jiang T, Xu K, Xie Y, Kim O, et al. Regulation of androgen receptor activity by tyrosine phosphorylation. Cancer Cell 2006;10: 309-19.

31. McCall P, Gemmell LK, Mukherjee R, Bartlett JM, Edwards J. Phosphorylation of the androgen receptor is associated with reduced survival in hormone-refractory prostate cancer patients. $\mathrm{Br} \mathrm{J}$ Cancer 2008;98:1094-101.

32. Ura S, Masuyama N, Graves JD, Gotoh Y. MST1-JNK promotes apoptosis via caspase-dependent and independent pathways. Genes Cells 2001;6:519-30.

33. Heemers HV, Tindall DJ. Androgen receptor (AR) coregulators: a diversity of functions converging on and regulating the AR transcriptional complex. Endocr Rev 2007;28:778-808.

34. Hodgson MC, Astapova I, Cheng S, Lee LJ, Verhoeven MC, Choi E, et al. The androgen receptor recruits nuclear receptor CoRepressor $(\mathrm{N}-\mathrm{CoR})$ in the presence of mifepristone via its $\mathrm{N}$ and $\mathrm{C}$ termini revealing a novel molecular mechanism for androgen receptor antagonists. J Biol Chem 2005;280:6511-9.

35. Liao G, Chen LY, Zhang A, Godavarthy A, Xia F, Ghosh JC, et al Regulation of androgen receptor activity by the nuclear receptor corepressor SMRT. J Biol Chem 2003;278:5052-61.

36. Guenther MG, Barak O, Lazar MA. The SMRT and N-CoR corepressors are activating cofactors for histone deacetylase 3. Mol Cell Biol 2001;21:6091-101. 
37. Zeng $\mathrm{Q}$, Hong W. The emerging role of the hippo pathway in cell contact inhibition, organ size control, and cancer development in mammals. Cancer Cell 2008;13:188-92.

38. Powzaniuk M, McElwee-Witmer S, Vogel RL, Hayami T, Rutledge SJ, Chen $\mathrm{F}$, et al. The LATS2/KPM tumor suppressor is a negative regulator of the androgen receptor. Mol Endocrinol 2004;18:2011-23.

39. Lee JH, Kim TS, Yang TH, Koo BK, Oh SP, Lee KP, et al. A crucial role of WW45 in developing epithelial tissues in the mouse. EMBO J 2008;27:1231-42.

40. Scher HI, Sawyers CL. Biology of progressive, castration-resistant prostate cancer: directed therapies targeting the androgen-receptor signaling axis. J Clin Oncol 2005;23:8253-61.
41. Mohler JL. Castration-recurrent prostate cancer is not androgenindependent. Adv Exp Med Biol 2008;617:223-34.

42. Edwards J, Krishna NS, Witton CJ, Bartlett JM. Gene amplifications associated with the development of hormone-resistant prostate cancer. Clin Cancer Res 2003;9:5271-81.

43. Ghosh PM, Malik SN, Bedolla RG, Wang Y, Mikhailova M, Prihoda TJ, et al. Signal transduction pathways in androgen-dependent and -independent prostate cancer cell proliferation. Endocr Relat Cancer 2005;12:119-34.

44. King JC, Xu J, Wongvipat J, Hieronymus $\mathrm{H}$, Carver BS, Leung DH, et al. Cooperativity of TMPRSS2-ERG with PI3-kinase pathway activation in prostate oncogenesis. Nat Genet 2009;41:524-6. 


\section{Cancer Research}

\section{MST1 Is a Multifunctional Caspase-Independent Inhibitor of Androgenic Signaling}

Bekir Cinar, Filiz Kisaayak Collak, Delia Lopez, et al.

Cancer Res 2011;71:4303-4313. Published OnlineFirst April 21, 2011.

$\begin{array}{ll}\text { Updated version } & \text { Access the most recent version of this article at: } \\ \text { doi:10.1158/0008-5472.CAN-10-4532 }\end{array}$

Supplementary Access the most recent supplemental material at:

Material http://cancerres.aacrjournals.org/content/suppl/2011/04/21/0008-5472.CAN-10-4532.DC1

Cited articles This article cites 44 articles, 20 of which you can access for free at:

http://cancerres.aacrjournals.org/content/71/12/4303.full\#ref-list-1

Citing articles This article has been cited by 2 HighWire-hosted articles. Access the articles at:

http://cancerres.aacrjournals.org/content/71/12/4303.full\#related-urls

E-mail alerts Sign up to receive free email-alerts related to this article or journal.

Reprints and To order reprints of this article or to subscribe to the journal, contact the AACR Publications

Subscriptions Department at pubs@aacr.org.

Permissions To request permission to re-use all or part of this article, contact the AACR Publications Department at permissions@aacr.org. 